\title{
El interés de la audiencia por las intervenciones televisivas de Pablo Iglesias: estrategia comunicativa de Podemos ${ }^{1}$
}

\author{
Jorge GALLARDO-CAMACHO \\ Universidad Camilo José Cela \\ jgallardo@ucjc.edu \\ Eva LAVÍN \\ Universidad Camilo José Cela \\ elavin@ucjc.edu
}

Recibido: 6 octubre de 2015

Aceptado: 19 de febrero de 2016

\begin{abstract}
Resumen
Este artículo analiza la eclosión mediática y política del partido político Podemos durante sus primeros meses de vida tras las elecciones europeas. Comprobaremos si el interés que despierta el partido en sus inicios, en intención de voto, se traslada a las audiencias de televisión con dos hipótesis que muestran la estrategia comunicativa de Podemos y su repercusión: la H1 afirma que los programas en los que participa Pablo Iglesias obtienen mayor audiencia de la habitual y la $\mathrm{H} 2$ plantea que la apuesta del líder de Podemos por el medio televisivo es muy superior y con mejores resultados con respecto a los partidos clásicos. Los resultados demuestran que los programas en los que participa $(\mathrm{N}=14)$ crecen un $62 \%$ y baten récord de audiencia. Concluimos que la estrategia comunicativa de Podemos da importancia a la televisión, además de continuar con la estrategia en redes aprendida en el 15M.
\end{abstract}

Palabras clave: Televisión, audiencias, comunicación política, cuota de pantalla, programación informativa, campañas, movimientos sociales, cadenas de televisión.

\section{The interest of the audience for the television appearances of Pablo Iglesias: communication strategy of Podemos}

\begin{abstract}
This paper analyzes the mediatic and political emergence of the political party Podemos during its firt months of life after the European elections. We will demonstrate the parallelism between the voting intentions and the television audiences with two hypothesis about the communication strategy of Podemos and its impact: H1 says that TV programs where Pablo Iglesias participates get more audience than usual and $\mathrm{H} 2$ affirms that the Podemos's leader strategy is focused on television with better results than classic parties. The results show that programs where Pablo Iglesias participates $(\mathrm{N}=14)$ grew $62 \%$, beating record audiences. We conclude that the communication strategy of Podemos gives importance to television with a big impact, besides going on with the strategy learned in the $15 \mathrm{M}$ in social networks.

Keywords: Television, audiences, political communication, share, news programming, political campaign, social movements, television channels.

1 Esta investigación es el resultado del proyecto de investigación "Análisis del impacto de las redes sociales y la construcción y credibilidad del discurso narrativo e informativo y audiovisual" subvencionado por la III Convocatoria de Ayudas a la Investigación Competitiva de la Universidad Camilo José Cela. Agradecemos a Kantar Media la facilitación de los datos necesarios para la realización de esta investigación.
\end{abstract}




\section{Referencia normalizada}

GALLARDO-CAMACHO, Jorge y LAVÍN, Eva (2016): "El interés de la audiencia por las intervenciones televisivas de Pablo Iglesias: estrategia comunicativa de Podemos". Estudios sobre el Mensaje Periodístico. Vol. 22, Núm. 1 (enero-junio), págs.: 273-286. Madrid, Ediciones Complutense.

Sumario: 1. Introducción; 1.1. La importancia de la audiencia televisiva en política; 1.2. La experiencia previa de Podemos con los medios; 1.3. Hipótesis de la investigación. 2. Metodología; 2.1. El interés social por Pablo Iglesias: sondeos y estimación de voto. 3. Resultados; 3.1. El incremento de audiencias y la contextualización de la estimación de voto; 3.2. Pablo Iglesias y los líderes de los partidos clásicos en televisión. 4. Discusión y conclusiones. 5. Referencias bibliográficas.

\section{Introducción}

En esta investigación vamos a analizar las apariciones del secretario general de Podemos, Pablo Iglesias, en la televisión tras irrumpir en el escenario político en España. Para comprender el análisis de audiencias que proponemos primero es necesario conocer el contexto socioeconómico en el que se produce (Orozco, 2001; Vela, 2001). En enero de 2014 surge un nuevo partido político denominado Podemos que en poco tiempo consigue posicionarse como una alternativa que desestabiliza el bipartidismo del Partido Popular (PP) y el Partido Socialista Obrero Español (PSOE). En este sentido, según Capilla (2014: 26) "Podemos ha protagonizado la irrupción más espectacular de una fuerza política desde el final de la dictadura" a través de un partido impulsado por un grupo de profesores de la facultad de Ciencias Políticas de la Universidad Complutense con el objetivo de acumular el voto de izquierda descontento con los partidos clásicos. Tras las manifestaciones y movimientos sociales del 15-M en España en 2011, Podemos ha sabido canalizar el malestar social hacia los partidos clásicos por los casos de corrupción, las altas tasas de paro y la precariedad laboral (Capilla, 2014; Ruiz, 2014). Por tanto, Urquizu (2015) añade que es imposible entender el fenómeno de Podemos en España sin saber lo que significó el movimiento de los indignados.

El 17 de enero de 2014 Podemos presentó su candidatura públicamente en Madrid tras haber mandado tres días antes el manifiesto "Mover ficha: convertir la indignación en cambio político" (Monedero et al. 2014) a los medios de comunicación. Con solo cuatro meses de vida, Podemos concurre a las elecciones europeas del 25 de mayo de 2014 y obtiene 1.245 .948 votos (cinco eurodiputados) convirtiéndose, de manera inesperada, en la cuarta fuerza política española en el Parlamento Europeo (2014). Iñigo Errejón (2014a; 2014b), jefe de campaña y el número dos de Podemos, analiza en varios artículos académicos lo que para ellos es una victoria.

La irrupción de Podemos ha revolucionado la forma de hacer marketing político con un posicionamiento cuidadosamente diseñado y una inteligente estrategia comunicativa para crear una marca poderosa (Ocaña, 2015). Por ejemplo, una de las decisiones que tomó Podemos en las elecciones europeas de 2014, inédita en España, fue la de poner la fotografía de su líder Pablo Iglesias en la papeleta para votar. Se observa por tanto que el fenómeno Podemos se personaliza en sus inicios en un líder y en "un hito cimentado en el carisma televisivo de Pablo Iglesias" (Capilla, 2014: 26). No obstante, cuando un partido tiene mayor recorrido histórico, las estrategias de los gru- 
pos políticos para ganar votos pueden estar asociadas con su imagen en conjunto y no necesariamente centrados en el candidato (Dias, 2005). No es nuestro caso ya que se trata de un partido que surge y crece rápidamente en pocos meses.

\subsection{La importancia de la audiencia televisiva en política}

Los estudios que analizan las apariciones concretas en televisión del líder de un partido emergente son escasos. Sin embargo, queda patente que la televisión es el medio escogido por la clase política para vender sus propuestas (Rodríguez \& Hernández, 2011). Según Laguna (2011) los asesores políticos se concentran más en la competencia mediática que en captar y formar militantes. Además afirma que las estrategias de los medios en su afán por ganar audiencia acrecienta este fenómeno, entrando en la personalización de la política. Sí existen estudios que aseguran que los debates políticos televisados con contertulios y políticos pueden influir sobre la preferencia por el voto por su poder persuasivo (Benoit, William, Hansen, \& Verser, 2003; Berrocal, 2005; Vázquez, 2005). Tras el primer debate televisado entre Richard Nixon y John F. Kennedy en 1960 la política cambió para siempre poniéndose de relieve la importancia de la telegenia (Ureña, 2008; Webley, 2010). Este debate fue el primer antecedente de la "vídeo-política" planteada por Sartori (1998: 66) como el poder del vídeo y "su incidencia en los procesos políticos". A partir de ese momento, se vio que era un requisito clave que el líder tuviera telegenia para así poder seducir al electorado a través de la pantalla (Laguna, 2011). Pero este estudio se relaciona con los investigadores que establecen que "la televisión es un medio que se nutre de agregar televidentes" (Landi, 1991: 206).

En España las primeras entrevistas políticas televisadas se realizaron en la campaña electoral de 1982 durante las segundas elecciones generales de la democracia. Estas se hicieron habituales en 1990 a raíz de la aparición de las televisiones privadas (RocaCuberes, 2013). De hecho, una encuesta concluye que la televisión continúa siendo el medio más influyente en el elector con un 34\% frente al 17\% de Internet (Abundis, 2015). Por su parte, el $56 \%$ de los españoles prefiere informarse a través de la televisión y el 48,6\% declara que este medio le influye mucho o bastante a la hora de decidir a quién votar (CIS, 2013). Por tanto, la televisión cambia la política aún en nuestros tiempos de digitalización de la comunicación (Matthes, 2012; Sartori, 2008).

\subsection{La experiencia previa de Podemos con los medios}

El origen de Podemos se sustenta en el movimiento $15 \mathrm{M}$ porque el equipo de campaña del nuevo partido político había aprendido de esa experiencia. El movimiento $15 \mathrm{M}$ se convirtió en un actor nuevo del sistema político español que influyó en las elecciones municipales de mayo de 2011 porque se trataba de un "movimiento contrahegemónico" (Errejón, 2011: 140). El campamento de la Puerta del Sol en Madrid (el núcleo físico del 15M donde se concentraron los manifestantes) contaba con medios de difusión en Internet: una televisión que llegó a recibir más de 11 millones de visitas, una radio (AgoraSol), un periódico que se podía descargar gratuitamente, además de contar con tres blogs y la Comisión de Difusión en Red que se encargaba de las redes sociales Twitter y Facebook (Mora: 2012). El Movimiento 15M es el último ejemplo de 
una serie de experiencias de movilización surgidas a partir de protestas convocadas mediante el uso de las nuevas tecnologías de la información y la comunicación (TIC) o que han destacado por el uso intensivo de las mismas (Díaz \& Candón, 2014: 463).

La experiencia directa con los medios de comunicación de los fundadores de Podemos la encontramos con "La Tuerka" (Castañón, 2014). Se trata de un programa amateur de debate político creado en el año 2010 por un grupo de personas relacionadas con la Facultad de Ciencias Políticas y Sociología de la Universidad Complutense de Madrid. Primero se emitía en la red de televisiones locales de la Asociación de Televisiones Locales de la Comunidad de Madrid y, actualmente, se emite en el diario digital Público. Pablo Iglesias es el presentador de un programa de entrevistas llamado "Otra vuelta de tuerka" que se difunde en directo y bajo demanda en la web. En este sentido, sus experiencias previas en "La Tuerka" les llevó a conclusiones como que su publicación en el portal Youtube les generaba un impacto importante cuando aseguran que "analizamos cómo ciertos cortes de vídeo de dos minutos triunfan y los ven diez veces más personas respecto a aquellos que ven un programa completo" (Errejón 2014b: 24). Pero lo que plantearemos en esta investigación es si Podemos va más allá e intenta extender su estrategia comunicativa a medios tradicionales como la televisión.

La primera campaña de Podemos se hizo con una veintena de personas aunque tiene especial relevancia el papel de su líder en la televisión como colaborador. Todo sucede en un contexto donde el mundo digital e Internet crecen en consumo aunque aún "se constata el dominio de la televisión" en el conjunto de la sociedad española (Callejo 2015: 12). Un año antes de las elecciones europeas, Pablo Iglesias empieza a presentar "Fort Apache", un programa emitido por el canal público iraní Hispan TV. Ese mismo año, en 2013, Iglesias empieza a tener apariciones en televisiones nacionales. Primero, en Intereconomía y después en cadenas con más audiencia como Cuatro; aunque llegará al horario de máxima audiencia como contertulio de "La Sexta Noche”. El formato de tertulia política es un género más informal que el debate y está asentado en la televisión española donde los contertulios combinan información y opinión (León \& Gómez, 2011). En este sentido, queda patente que el líder político que no consiga reproducir sus ideas desde los códigos televisivos pasará desapercibido para la opinión pública (Rodríguez \& Hernández, 2011). Con esta introducción hemos conocido los estudios que contextualizan y justifican el objeto de estudio: el análisis de audiencias de Pablo Iglesias en televisión.

\subsection{Hipótesis de la investigación}

Planteamos dos hipótesis de investigación que se complementan entre sí. Hipótesis 1 (H1): los programas de televisión en los que interviene Pablo Iglesias en prime time tienen una audiencia superior a la habitual, lo que reflejaría el interés del espectador por las palabras del líder de Podemos. Hipótesis 2 (H2): el medio televisivo en horario de máxima audiencia es más importante para Pablo Iglesias como estrategia comunicativa con respecto a los líderes de los dos grandes partidos clásicos (PSOE y PP) y obtiene mejores resultados de audiencia que estos. Por tanto, nuestro objetivo general es conocer la estrategia de comunicación de Podemos con respecto a la televisión tradicional y su repercusión entre los espectadores. 


\section{Metodología}

Para la comprobación de las hipótesis recurriremos a una metodología principalmente cuantitativa a partir de la recopilación y posterior comparación de los datos de audiencia. El estudio se centra en las intervenciones de Pablo Iglesias en televisiones españolas de ámbito estatal y en el horario de máxima audiencia durante un ámbito de análisis de 11 meses que abarca el periodo establecido desde la celebración de las elecciones europeas (25 de mayo de 2014) hasta las siguientes elecciones autonómicas en Andalucía (21 de marzo de 2015). Es relevante la elección de este periodo ya que refleja la etapa de crecimiento en las encuestas de Podemos en su fase de eclosión. Además, creemos que es un periodo suficiente para alcanzar resultados de interés de cara a la confirmación o no de las hipótesis; por otra parte, es importante delimitar la participación del líder de Podemos en los programas de televisión en horario de máxima audiencia ya que el perfil del espectador está menos condicionado por los rasgos específicos de una franja determinada como podría ser el horario de mañana (donde el espectador es de mayor edad y hay más mujeres que hombres). El prime time en España se denomina "Noche 1" y ocupa la franja horaria que comienza aproximadamente entre las nueve de la noche y la medianoche (Barlovento Comunicación, 2015a).

Los datos han sido extraídos y calculados a través de la aplicación Infosys (2014) de la empresa que cuantifica oficialmente la audiencia televisiva en España: Kantar Media. Por su parte, la metodología basada en la comparación de los datos de share (cuota de pantalla) está basada en la realizada por la consultora de investigación Barlovento Comunicación (2015a, 2015b). Sin embargo, para poder detectar los programas de televisión en los que ha intervenido Pablo Iglesias ha sido necesario recurrir a la observación íntegra de las emisiones de los programas de actualidad informativa en los que potencialmente podía haber sido entrevistado. Una vez tomada la frecuencia de aparición como uno de los criterios de clasificación (Pinto \& Grawitz, 1967), se recurrió a la extracción de datos de esos programas para la elaboración de los resultados. En ese sentido, hemos obtenido una muestra de 14 intervenciones de Pablo Iglesias.

Para la comprobación de la H1, la información que hemos recopilado para elaborar la organización de los datos en tablas han sido: nombre del programa, tipo de participación (entrevista en plató, contertulio o conexión en directo), fecha de emisión y cadena de emisión. Posteriormente, hemos procedido a la extracción de datos relacionados con las audiencias a partir de estas variables: audiencia media del programa (en función del dato de cuota de pantalla denominado share), número de espectadores medio, posición del programa entre los 15 programas más vistos del día (según el share), posición del programa en su franja de emisión entre 15 emisiones (según el share), audiencia media del programa durante la temporada 2014-2015 (según el share y hasta la fecha del último programa emitido durante el periodo de estudio). Para la comprensión de los resultados hay que tener en cuenta que las televisiones más vistas en España según su cuota de pantalla en el mes de la última intervención registrada de Pablo Iglesias son las siguientes (Barlovento Comunicación, 2015b): Telecinco $(15,4 \%)$, Antena $3(13,2 \%)$, La $1(10,2 \%)$, La Sexta $(8 \%)$ y Cuatro $(7,5 \%)$. Con res- 
pecto a la $\mathrm{H} 2$, también se registró el porcentaje de share, el número de espectadores y la frecuencia de las entrevistas a Mariano Rajoy (PP) y Pedro Sánchez (PSOE) durante el periodo de análisis para establecer una posterior comparación. Como todas las muestras se corresponden al mismo programa de televisión ("Informativos Telecinco") la comparación entre los datos permitirá resultados más objetivos.

Debemos explicar que tendremos en cuenta la participación de Pablo Iglesias en algunos programas de la muestra como contertulio (y no como entrevistado) ya que pensamos que es relevante analizar precisamente esa evolución tras las elecciones europeas. Por tanto, en las tablas expuestas en los resultados los datos de las casillas relacionadas con programas en los que Iglesias ha participado como contertulio son orientativos ya que comparte plató de televisión con otros colaboradores y es difícil asegurar que el interés por su Figura ha incrementado la audiencia (por este motivo tampoco se ha establecido el periodo de análisis antes de las elecciones europeas).

\subsection{El interés social por Pablo Iglesias: sondeos y estimación de voto}

En la $\mathrm{H} 1$ planteamos que en caso de producirse un incremento de audiencia en las participaciones de Pablo Iglesias se debe al interés que despierta su líder y Podemos en la sociedad durante el periodo de estudio. Creemos que una forma de reflejar ese interés de la población por el nuevo partido es a través de los sondeos. Pablo Iglesias se convierte según el sondeo de Sigma Dos (Méndez, 2014) de noviembre de 2014 en el líder mejor valorado en España $(4,37)$ por delante de Pedro Sánchez del PSOE $(4,12)$ y el presidente Mariano Rajoy del PP $(3,5)$. Pero hay otro indicador también de interés que es el barómetro de voto del Centro de Investigaciones Sociológicas (CIS, 2015) que sitúa a Podemos en constante crecimiento en estimación de voto desde las elecciones europeas en mayo de 2014 hasta convertirse en menos de un año en la segunda fuerza política en el periodo de estudio. Si nos basamos en la investigación de Humanes (2014) observamos además que existe una exposición selectiva de medios en función de la ideología de la audiencia que podría trasladarse a nuestro estudio aunque observa que esa vinculación es más fuerte en la prensa escrita que en la televisión. En este sentido, plantearemos una comparativa entre la evolución de la audiencia y la estimación de voto en el periodo de estudio con el programa que más frecuencia tiene en los resultados, sobre todo, con fines de contextualización (ya que haría falta un periodo de análisis más amplio para encontrar una correlación significativa). En este sentido, Martín y Álvarez de Arcaya (2003) analizan las audiencias en política e invitan a explorar métodos alternativos a las encuestas de opinión para mediar el interés hacia la información política.

\section{Resultados}

Tras observar las emisiones en las que potencialmente podría haber intervenido Pablo Iglesias localizamos 14 programas de televisión, como vemos en la Tabla 1. 
Tabla 1. Los datos de audiencia de los programas de la muestra con respecto a la competencia (en negrita: las emisiones más vistas con respecto a los programas de la temporada). Fuente: elaboración propia a partir de la extracción de datos de Kantar Media. *En negrita: las emisiones que han sido más vistas con respecto a todos los programas de la temporada.

\begin{tabular}{|l|c|c|c|r|r|r|r|}
\hline \multicolumn{1}{|c|}{ Programa } & Cadenas & Fecha & Participación & $\begin{array}{c}\text { Share } \\
\text { programa }\end{array}$ & $\begin{array}{c}\text { No } \\
\text { espectadores }\end{array}$ & $\begin{array}{c}\text { Posición en } \\
\text { ranking más } \\
\text { vistos día }\end{array}$ & $\begin{array}{c}\text { Posición } \\
\text { en franja }\end{array}$ \\
\hline 1. La Sexta Noche & La Sexta & $31 / 05 / 14$ & Contertulio & $15,2 \%$ & 2.087 .000 & 3 & 2 \\
\hline 2. El Objetivo & La Sexta & $01 / 06 / 14$ & Entrevista & $12,5 \%$ & 2.482 .000 & 6 & 3 \\
\hline 3. La Sexta Noche & La Sexta & $14 / 06 / 14$ & Contertulio & $11,2 \%$ & 1.337 .000 & 13 & 4 \\
\hline 4. La Sexta Noche & La Sexta & $05 / 07 / 14$ & Contertulio & $12,0 \%$ & 1.251 .000 & 13 & 3 \\
\hline 5. La Sexta Noche & La Sexta & $19 / 07 / 14$ & Contertulio & $15,9 \%$ & 1.483 .000 & 10 & 2 \\
\hline $\begin{array}{l}\text { 6. Viajando con } \\
\text { Chester }\end{array}$ & Cuatro & $28 / 09 / 14$ & Entrevista & $\mathbf{1 4 , 5 \%}$ & $\mathbf{2 . 8 2 3 . 0 0 0}$ & 3 & 1 \\
\hline 7. La Sexta Noche & La Sexta & $04 / 10 / 14$ & Entrevista & $\mathbf{1 6 , 2 \%}$ & 2.015 .000 & 2 & 2 \\
\hline 8. Salvados & La Sexta & $26 / 10 / 14$ & Entrevista & $\mathbf{2 3 , 8 \%}$ & $\mathbf{4 . 9 4 2 . 0 0 0}$ & 1 & 1 \\
\hline $\begin{array}{l}\text { 9. La Sexta } \\
\text { Columna }\end{array}$ & La Sexta & $07 / 11 / 14$ & Entrevista & $\mathbf{1 3 , 8 \%}$ & $\mathbf{2 . 4 5 5 . 0 0 0}$ & 3 & 1 \\
\hline 10. El Objetivo & La Sexta & $16 / 11 / 14$ & Entrevista & $\mathbf{1 4 , 6 \%}$ & $\mathbf{3 . 0 3 1 . 0 0 0}$ & 3 & 2 \\
\hline $\begin{array}{l}\text { 11. La noche en } \\
\text { 24H }\end{array}$ & Canal 24H & $05 / 12 / 14$ & Entrevista & $\mathbf{3 , 1 \%}$ & $\mathbf{4 9 8 . 0 0 0}$ & - & - \\
\hline $\begin{array}{l}\text { 12. La Sexta } \\
\text { Noche }\end{array}$ & La Sexta & $24 / 01 / 15$ & Entrevista & $16,0 \%$ & $\mathbf{2 . 1 0 7 . 0 0 0}$ & 8 & 2 \\
\hline 13. El Objetivo & La Sexta & $25 / 01 / 15$ & Conex. Directo & $10,7 \%$ & 2.284 .00 & 10 & 4 \\
\hline $\begin{array}{l}\text { 14. Informativos } \\
\text { T5 }\end{array}$ & Telecinco & $23 / 02 / 15$ & Entrevista & $\mathbf{2 2 , 2 \%}$ & $\mathbf{4 . 2 6 9 . 0 0 0}$ & 2 & 1 \\
\hline & & & PROMEDIO & $\mathbf{1 4 , 4 \%}$ & $\mathbf{2 . 3 6 7 . 6 9 2}$ & $\mathbf{5 , 9}$ & $\mathbf{2 , 1}$ \\
\cline { 5 - 8 }
\end{tabular}

En cuanto al modo de participación, tras las elecciones europeas, observamos que la primera intervención del líder de Podemos es como contertulio. Después lo hará como entrevistado en el programa "El Objetivo", pero las tres siguientes ocasiones también seguirá como contertulio hasta continuar siempre como entrevistado.

En la Tabla 1 vemos que de los siete programas diferentes en los que participa Iglesias, todos baten récord de audiencia de temporada según su share: "La Sexta Noche", "El Objetivo", "Viajando con Chester", "La Sexta Columna", "La noche en 24H", "Salvados" e "Informativos Telecinco". En primer lugar, "La Sexta Noche" aparece en seis ocasiones en la muestra y en dos de ellas bate récord con respecto a las 110 emisiones desde su primera temporada en el año 2013 (la emisión del 4 de octubre de 2014 consigue el mayor share histórico con un 16,2\% y el 21 de enero de 2015 bate récord de espectadores). Por su parte, "El Objetivo" (también en La Sexta) consigue el mejor share de la temporada y el segundo mejor share de su historia (con respecto a sus 60 emisiones hasta febrero de 2015) con la entrevista a Pablo Iglesias el 16 de noviembre de 2014. "La Sexta Columna" también obtiene su mejor share de la historia desde el año 2012 (entre 99 programas) con el programa dedicado a Pablo Iglesias con un 13,8\%. El canal temático Canal 24 Horas bate récord histórico con un 3,1\% de share. Y, por último, "Viajando con Chester" también se convierte en la emisión más vista de la temporada y de su historia con un 14,6\% (entre 26 programas emitidos). 
"Informativos Telecinco" congregó a 4.269.000 espectadores con un 22,2\% de cuota de pantalla convirtiéndose en el informativo más visto de la historia de Mediaset y en la entrevista política más vista en España desde el año 2004 cuando se entrevistó, también en Telecinco, a José María Aznar obteniendo un 31,5\% de share con 5.719.000 espectadores (Barlovento Comunicación, 2015b). En cuanto al programa "Salvados", la entrevista a Pablo Iglesias es el segundo programa más visto de entre sus 201 emisiones desde 2008 y el más visto de la temporada hasta febrero de 2015.

En cuanto a la posición en el ranking de los programas más vistos de cada día, en todos los casos ha estado al menos entre las 13 emisiones más vistas y con una media con la que ocuparía la quinta posición (la cuarta posición si $\mathrm{N}=9$ descartando los programas en los que es contertulio y el "Canal 24 horas" del que no hay datos al estar más allá de las 14 emisiones más vistas). De los 14 programas analizados, tan solo una vez ha sido el programa más visto del día y la mitad de las veces ha estado entre los tres primeros. Con respecto a su competencia directa (en su franja de emisión) el programa donde ha aparecido Pablo Iglesias ha sido líder en cuatro ocasiones (posiciones 6, 8, 9 y 14 en la Tabla 1) y ocupa la segunda plaza como promedio de todas las emisiones analizadas en el estudio (promedio de posición 2,1 para $\mathrm{N}=14$; y 1,9 para $\mathrm{N}=9$ ). Si nos centramos en los datos de audiencia de la Tabla 1, el share y el número de espectadores nos servirán de referencia para comparar las cifras en posteriores tablas. De esta manera, en la Tabla 2 cuantificamos porcentualmente el incremento de audiencia que se produce en los programas en los que interviene Pablo Iglesias.

Tabla 2. Incremento porcentual de audiencia en los programas en los que participa Pablo Iglesias. Fuente: elaboración propia a partir de la extracción de datos de

Kantar Media.

\begin{tabular}{|l|r|r|r|r|}
\hline $\begin{array}{l}\text { Programa por orden de } \\
\text { emisión }\end{array}$ & $\begin{array}{l}\text { Share } \\
\text { Programa, SP } \\
\text { (con Pablo } \\
\text { Iglesias) }\end{array}$ & $\begin{array}{l}\text { Share } \\
\text { Temporada del } \\
\text { programa, ST }\end{array}$ & $\begin{array}{l}\text { Diferencia } \\
\text { (SP - ST) }\end{array}$ & $\begin{array}{l}\text { \% incremento del } \\
\text { share en programas } \\
\text { con Pablo Iglesias }\end{array}$ \\
\hline 1. La Sexta Noche & $15,2 \%$ & $10,5 \%$ & $4,7 \%$ & $+44,8 \%$ \\
\hline 2. El Objetivo & $12,5 \%$ & $9,0 \%$ & $3,5 \%$ & $+38,9 \%$ \\
\hline 3. La Sexta Noche & $11,2 \%$ & $10,5 \%$ & $0,7 \%$ & $+6,7 \%$ \\
\hline 4. La Sexta Noche & $12,0 \%$ & $10,5 \%$ & $1,5 \%$ & $+14,3 \%$ \\
\hline 5. La Sexta Noche & $15,9 \%$ & $10,5 \%$ & $5,4 \%$ & $+51,4 \%$ \\
\hline 6. Viajando con Chester & $14,5 \%$ & $8,3 \%$ & $6,2 \%$ & $+74,7 \%$ \\
\hline 7. La Sexta Noche & $16,2 \%$ & $10,5 \%$ & $5,7 \%$ & $+54,3 \%$ \\
\hline 8. Salvados & $23,8 \%$ & $14,3 \%$ & $9,5 \%$ & $+66,4 \%$ \\
\hline 9. La Sexta Columna & $13,8 \%$ & $7,9 \%$ & $5,9 \%$ & $+74,7 \%$ \\
\hline 10. El Objetivo & $14,6 \%$ & $9,0 \%$ & $5,6 \%$ & $+62,2 \%$ \\
\hline 11. La noche en 24H & $3,1 \%$ & $0,8 \%$ & $2,3 \%$ & $+287,5 \%$ \\
\hline 12. La Sexta Noche & $16,0 \%$ & $10,5 \%$ & $5,5 \%$ & $+52,4 \%$ \\
\hline 13. El Objetivo & $10,7 \%$ & $9,0 \%$ & $1,7 \%$ & $+18,9 \%$ \\
\hline 14. Informativos T5 & $22,2 \%$ & $18,4 \%$ & $3,8 \%$ & $+20,7 \%$ \\
\hline PROMEDIO & $\mathbf{1 4 , 4 \%}$ & $\mathbf{1 0 , 0 \%}$ & $\mathbf{4 , 4 \%}$ & $+\mathbf{6 2 , 0 \%}$ \\
\hline
\end{tabular}


En la Tabla 2 se observa si existe un incremento de cuota de pantalla de las emisiones concretas en las que participa Pablo Iglesias con respecto al dato de share medio de ese programa durante la temporada televisiva de estudio. En todos los programas de la muestra $(\mathrm{N}=14)$ se produce un incremento y la diferencia media $(\mathrm{SP}-\mathrm{ST})$ es del $4,4 \%$ en su share con respecto a los programas emitidos en la misma temporada. Es decir, el crecimiento porcentual medio de audiencia en los programas en los que aparece Pablo Iglesias es de un $62 \%$ (y un $75 \%$ si $N=10$, excluyendo los programas en los que fue contertulio). El programa que más crece es "La Noche en 24 horas" que pasa de un $0,8 \%$ de share a un 3,1\% (lo que supone un crecimiento porcentual de un 287,5\%). El programa que menos crece es una de las emisiones de "La Sexta Noche" (posición 3 de la Tabla 3) que apenas tiene una variación positiva de $0,7 \%$ de share con respecto a la media. No obstante, los 6 programas de "La Sexta Noche" (programas 1, 3, 4, 5, 7 y 12 por orden de emisión) tienen un incremento medio porcentual de un 37,3\%.

\subsection{El incremento de audiencias y la contextualización de la estimación de voto} Hemos decidido centrarnos en el programa que más frecuencia tiene en los resultados expuestos: "La Sexta Noche". En la Figura 1, solo con fines de contextualización, analizamos la línea de tendencia del porcentaje de estimación de votos del CIS (2014a, 2014b, 2014c, 2015) y del resultado de las elecciones europeas del mes de mayo (Parlamento Europeo, 2014) con respecto al promedio de espectadores que ven a Pablo Iglesias.

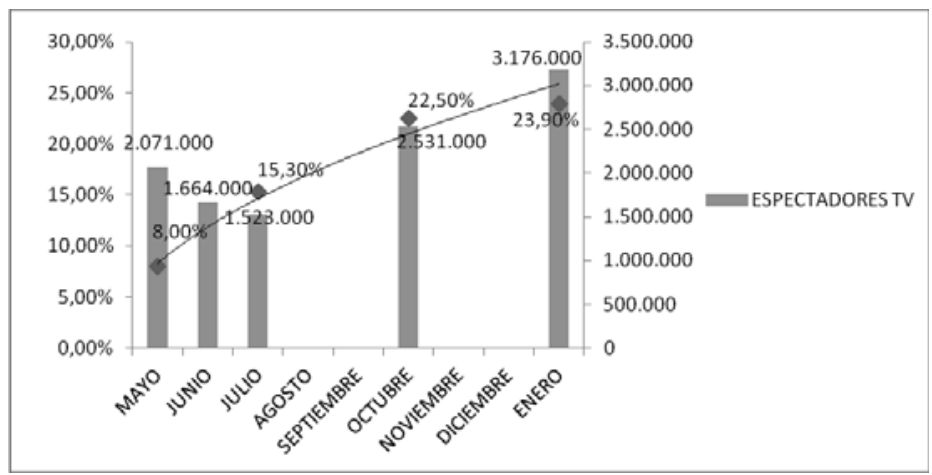

Figura 1. Comparación del voto a Podemos y espectadores de televisión de "La Sexta Noche" (Elaboración propia)

De esta manera, el punto con mayor estimación de voto lo encontramos en el mes de enero de $2015(23,9 \%)$ y coincide con el máximo de espectadores (3.176.000).

\subsection{Pablo Iglesias y los líderes de los partidos clásicos en televisión}

Durante el periodo de estudio los líderes del PP y PSOE concedieron una sola entrevista en horario de máxima audiencia en televisión en el mismo espacio en el que también es entrevistado Pablo Iglesias: "Informativos Telecinco" (programa 14, tablas 1 y 2). Pablo Iglesias concedió 10 entrevistas y participó en 4 programas como contertulio. Eso nos facilita establecer una comparación cuantitativa entre los resultados obtenidos por cada líder. 
La entrevista al presidente popular Mariano Rajoy (emitida en directo el 26 de enero de 2015) obtuvo un 19,2\% de share y 3.647 .000 espectadores. Dos semanas después (9 de febrero de 2015) es entrevistado el líder del PSOE, Pedro Sánchez, cuya audiencia fue de un $16,4 \%$ y 3.117 .000 espectadores. Por su parte, la entrevista a Pablo Iglesias en "Informativos Telecinco" se hizo el 22 de febrero de 2015 y congregó a 4.269.000 espectadores con un 22,2\%. Cuantitativamente la entrevista de Pablo Iglesias ha conseguido 3 puntos más de audiencia y 622.000 espectadores más que Mariano Rajoy; y 5,8 puntos más de share y 1.152 .000 más espectadores que Pedro Sánchez.

\section{Discusión y conclusiones}

Con respecto a la $\mathrm{H} 1$, se confirma que las intervenciones de Pablo Iglesias producen un incremento de audiencia en las 14 emisiones en las que participa según el share de la temporada analizada o incluso, en algunos casos, de toda su historia. Por su parte, Iglesias participa en 7 programas diferentes y en todos ellos otorga en alguna ocasión el récord de temporada; en el caso de "La Sexta Noche" le da récord de share (programa 7, Tabla 1) y de espectadores (programa 12, Tabla 1) en días diferentes. Los resultados demuestran pues que el interés por el fenómeno Podemos se traslada a una subida en las audiencias de televisión de los programas en los que participa el líder del nuevo partido político. No obstante, hemos visto que la intervención de Pablo Iglesias no asegura el liderazgo como el programa más visto en su franja o en el día. En ese sentido, es más probable, tras el análisis de la muestra, que si un programa entrevista a Pablo Iglesias sea líder en la franja (con un promedio de la segunda posición aproximadamente) en lugar de la emisión más vista del día (con un promedio de la cuarta plaza). Es decir, Pablo Iglesias aseguraría un crecimiento de audiencia pero no aportaría el liderazgo absoluto. Por tanto, el interés por Podemos se contextualiza durante el periodo de análisis como una tendencia ascendente según los datos del CIS que se ve reflejada también en los datos de audiencia (Figura 1). Observamos una línea de tendencia ascendente que va acompañada de un incremento de espectadores, exceptuando los meses de verano en los que hay que tener en cuenta que el consumo de televisión baja usualmente (Barlovento Comunicación, 2015b). No obstante, pensamos que sería de interés continuar analizando esa correlación en futuras investigaciones hasta que se produzca un descenso notable en la estimación de voto para comprobar si se traslada a un desinterés por parte de la audiencia televisiva.

En cuanto a la H2, se confirma que Pablo Iglesias supera de manera exponencial la presencia en televisión con respecto a Mariano Rajoy y Pedro Sánchez (14 intervenciones frente a una) además de obtener mayor número de espectadores y cuota de pantalla. La entrevista al líder de Podemos fue la emisión más vista del mes de febrero y de la historia de Telecinco (Barlovento Comunicación, 2015b). Hay que tener en cuenta además que la entrevista de Telecinco se produce cuando el último dato del CIS sobre la estimación de voto de Podemos es de un $23,9 \%$ convirtiéndose en la segunda fuerza política (CIS, 2015).

El cumplimiento de estas dos hipótesis, la demostración de las mismas y la contextualización del artículo nos han permitido observar la estrategia de comunicación de Podemos y obtener algunas conclusiones. Por ejemplo, el partido de Pablo Iglesias mantiene la estrategia de comunicación en redes sociales (aprendida durante la expe- 
riencia de sus miembros en el $15 \mathrm{M}$ ) como carácter diferenciador, pero añade una presencia excepcionalmente superior con respecto a los partidos clásicos en la televisión. En este caso, Pablo Iglesias sigue la afirmación de Rodríguez y Hernández (2011) sobre el surgimiento de un líder electrónico, un político capaz de adecuar su mensaje al medio televisivo para convencer y persuadir a la opinión pública. La demostración de las hipótesis confirma las palabras de Errejón (2014a, 2014b: 40) cuando asevera que la televisión es la más poderosa herramienta comunicativa de su campaña y que Podemos "sube la audiencia", al menos durante nuestro periodo de análisis.

Por otro lado, el partido empieza a dar importancia a la fuerza comunicativa de la televisión en el momento en el que Pablo Iglesias deja de acudir a los programas como colaborador y solo lo hace como entrevistado para ganar entidad como candidato a la presidencia del gobierno. Precisamente, solo acude como entrevistado a partir del mes en el que el CIS (2014b) establece que la estimación de voto de Podemos es de un $15 \%$. También es notable que la cadena de televisión en la que tiene más apariciones es en La Sexta (11 de 14); sorprende también que en el periodo de estudio no haya entrevistas a Pablo Iglesias en horario de máxima audiencia en dos grandes cadenas nacionales: La 1 (la principal cadena de Radio Televisión Española) y Antena 3 (del mismo grupo de comunicación que La Sexta). En este sentido, podría justificarse este hecho porque La Sexta tiene un "telespectador situado más hacia la izquierda ideológica y política" (Humanes, 2014: 790) y quizás Podemos y su líder se encuentren más cómodos y estimen que llegan más a sus espectadores. No obstante, también entendemos que el crecimiento vertiginoso de Podemos en el CIS podría generar un interés también en las personas contrarias a su ideología y eso también podría producir un crecimiento de audiencia; a pesar de que Hallin y Mancini (2004) insisten en que el sistema español de medios se caracteriza por audiencias partidistas. Pero aquí es donde reside la excepcionalidad del fenómeno de estudio. Es decir, los espectadores que ven a Pablo Iglesias en la televisión no tienen por qué estar desacuerdo con sus propuestas o su ideología, pero sí es cierto que puede despertar el interés tanto en sus simpatizantes como en sus detractores. El contexto socioeconómico ha creado una bolsa de población descontenta, indecisa en su voto e interesada en oír las propuestas de partidos emergentes como Podemos. Por tanto, la televisión como medio vuelve a demostrar que incrementa su audiencia cuando ofrece temas de interés general. En ese sentido, mientras Sartori (1998: 72) asegura que "la televisión refleja los cambios que promueve e inspira a largo plazo" pensamos que en nuestro caso de estudio se demuestra que a corto plazo también se pueden promover cambios.

Este estudio abre una nueva línea de investigación de un líder de un partido político emergente vinculado al estudio de audiencias y a la estrategia comunicativa. Sería de interés continuar estudiando los movimientos comunicativos de este partido emergente y de otros como Ciudadanos y analizar su relación con medios tradicionales como la televisión.

\section{Referencias bibliográficas}

ABUNDIS, Francisco (2015): "Internet, el medio que menos influye en las elecciones", en El Financiero, 18 de febrero: (http://goo.gl/f5kQVD) [Consulta: 22 de febrero de 2015]. 
BARLOVENTO COMUNICACIÓN (2015a): “Análisis Televisivo 2014”, 2 de enero de 2014: http://goo.gl/qy2Xdv [Consulta: 22 de agosto de 2015].

BARLOVENTO COMUNICACIÓN (2015b): "El comportamiento de la audiencia televisiva": http://goo.gl/FKGgj1 [Consulta: 20 de agosto de 2015].

BENOIT, William L., HANSEN, Glenn J. \& VERSER, Rebecca M. (2003). “A metaanalysis of the effects of viewing U.S. Presidential Debates". Communication Monographs, 70 (4), pp. 335-350. DOI: 10.1080/0363775032000179133

BERROCAL, Salomé (2005): "La información política en televisión: ¿apatía o interés entre los telespectadores?". Comunicar, 25, pp. 1-7: http://goo.g1/KTD9j4 [Consulta: 10 de agosto de 2015].

CALLEJO, Javier (2015): "Las dietas mediáticas de los españoles. Estudio a través de los usos del tiempo en 2009-2010". Revista Internacional de Sociología, 73 (1). DOI: http://dx.doi.org/10.3989/ris.2013.04.15

CAPILLA, Manuel (2014): "Los hombres clave de Podemos". El siglo de Europa, 1066, 26-28: http://goo.gl/50eeso [Consulta: 15 de agosto de 2015].

CASTAÑÓN, César (2014): "100 días, 100 años. La irrupción de PODEMOS en la crisis del régimen político español”. Kultur, vol. 1, 2, 155-170: http://goo.gl/S1r29v [Consulta: 01 de agosto de 2015].

CIS (Centro de Investigaciones Sociológicas) (2013): Barómetro de marzo. Estudio $\mathrm{n}^{\mathrm{o}}$ 2.981: http://goo.gl/YliQN1 [Consulta: 1 de agosto de 2015].

CIS (2014a): Barómetro de abril. Estudio no 3021: http://goo.gl/11O7dL [Consulta: 3 de agosto de 2015].

CIS (2014b): Barómetro de julio. Estudio n ${ }^{\mathrm{o}} 3033$ : http://goo.gl/NBKhec [Consulta: 1 de agosto de 2015].

CIS (2014c): Barómetro de octubre. Estudio no 3041: http://goo.gl/rlPUK4 [Consulta: 01 de agosto de 2015].

CIS (2015): Barómetro de enero. Estudio no 3050: http://goo.gl/q6g9G1 [Consulta: 1 de agosto de 2015].

DIAS, Marcia Ribeiro (2005): "Projeção da imagem partidária nas estratégias de campanha na televisão: uma análise do HGPE 2002". Dados, 48, 1. DOI: http://dx.doi.org/10.1590/S0011-52582005000100006

DÍAZ, Ibán \& CANDÓN, José (2014): "Espacio geográfico y ciberespacio en el movimiento 15M". Scripta Nova, 18, 463-499: http://goo.gl/sD3GOp [Consulta: 1 de septiembre de 2015].

ERREJÓN, Iñigo (2011): "El 15-M como discurso contrahegemónico". Encrucijadas, 2, 120-145: http://goo.gl/n1bBR1 [Consulta: 14 de agosto de 2015].

ERREJÓN, Iñigo (2014a): “¿Qué es Podemos?: terremoto en el escenario político", en Le Monde Diplomatique en español, 225, pp. 1-3: http://goo.gl/2O8j19 [Consulta: 12 de septiembre de 2015]. 
ERREJÓN, Iñigo (2014b): "PODEMOS como práctica cultural emergente frente al imaginario neoliberal: hegemonía y disidencia". IC Revista Científica de Información y Comunicación, 11, 17-44: http://goo.gl/TCbjpe [Consulta: 20 de agosto de 2015].

HALLIN, Daniel \& MANCINI, Paolo (2004): Comparing Media Systems. Three models of media and politics. New York, Cambridge University Press.

HUMANES, María Luisa (2014): "Exposición selectiva y partidismo de las audiencias en España". Palabra Clave, 17 (3), pp. 773-802. DOI: 10.5294 /pacla.2014.17.3.9

INFOSYS. (2014): [Programa informático]. InfoSys, version i+.

LAGUNA, Antonio (2011): "Liderazgo y Comunicación: La Personalización de la Política". Anàlisi, quaderns de comunicació i cultura. 43, pp. 45-57.

LANDI, Óscar. (1991): "La televisión y el futuro de la política". Comunicación y Sociedad, 10-11, pp. 193-208: http://goo.gl/9OYJPj [Consulta: 12 de agosto de 2015].

LANDRY, Rèjean. (1997): "L'analyse de contenu". En GAUTHIER, Benoît (Ed.), Recherche sociale. De la problemátique à la collecte des données, pp. 329-356. Sillery, Presses de l'Université du Québec.

LEÓN GROSS, Teodoro y GÓMEZ CALDERÓN, Bernardo (2011): "La tertulia en España: medios públicos, última frontera de la pluralidad”. Estudios sobre el Mensaje Periodístico, vol. 17, núm. 1, pp. 67-80. Madrid, Servicio de Publicaciones de la Universidad Complutense. DOI: http://dx.doi.org/10.5209/rev_ESMP.2011 .v17.n1.4

MARTÍN, Marta y ÁLVAREZ DE ARCAYA, Helena (2003): "Comunicación electoral e interés ciudadano". Zer, 14, pp. 75-96: http://goo.gl/DhwTQ9 [Consulta: 12 de septiembre de 2015].

MATTHES, Jörg (2012): "Exposure to counter attitudinal news coverage and the timing of voting decisions". Communication Research, 39 (2), pp. 147-169. DOI: $10.1177 / 0093650211402322$

MÉNDEZ, Lucía (2014): "Pablo Iglesias y Sánchez, los mejor valorados", en El Mundo, 24 de Noviembre de 2014: http://goo.gl/ly2PiZ [Consulta: 5 de agosto de 2015].

MONEDERO, José Carlos et al. (2014): "Mover ficha: convertir la indignación en cambio político": http://goo.gl/GdQKMK [Consulta: 12 de agosto de 2015].

MORA, Fernando (2012): “Organización y participación en el 15M". Praxis sociológica, 16, pp. 99-124.

OCAÑA, Silvia (2015): “La marca Podemos: expertos en planificación y marketing político analizan las claves de su estrategia comunicativa". Anuncios: Semanario de publicidad y marketing. 1503, pp. 36-38. 
OROZCO, Guillermo (2001): Televisión y audiencias: un enfoque cualitativo. Madrid, Ediciones de La Torre.

PARLAMENTO EUROPEO (2014): "Resultados de las elecciones europeas de 2014" (http://goo.gl/oNk0rA) (26-03-2014).

PINTO, Roger \& GRAWITZ, Madeleine (1967): Analyse de contenu et theorie. Méthodes des sciences sociales. Paris, Dalloz.

ROCA-CUBERES, Carles (2013): "Desarrollo histórico y actualidad de la entrevista política televisada en España". Historia y comunicación social, 18, 3, pp. 437-447. DOI: http://dx.doi.org/10.5209/rev_HICS.2013.v18.44340

RODRÍGUEZ, Imelda y HERNÁNDEZ, María (2011): “Análisis de la comunicación no verbal de José Luis Rodríguez Zapatero". Revista Latina de Comunicación Social, ${ }^{\circ} 65$, pp. 436-449. DOI: 10.4185/RLCS-65-2010-911-436-459

RUIZ, Domènec (2014): "El populismo de 'izquierda' en España: el caso de Podemos". Temas para el debate, 240, pp. 32-34: http://goo.gl/xdZuAp [Consulta: 12 de agosto de 2015].

SARTORI, Giovanni (1998): Homo videns. La sociedad teledirigida. Buenos Aires, Taurus.

SARTORI, Giovanni (2008): Elementos de Teoría Política. Madrid, Alianza Editorial.

UREÑA, Daniel (2008): "El ejemplo americano", en $A B C, 24$ de febrero de 2008: http://goo.gl/4APyYS (20-01-2015).

URQUIZU, Ignacio (2015): “15M y Podemos: ¿almas gemelas?”, en El País, 31 de enero de 2015: http://goo.gl/qmBj5S [Consulta: 10 de agosto de 2015].

VÁZQUEZ, Sandra (2005): "La televisión persuasiva". Comunicar, 13, p. 25: http://goo.gl/T1w0SL [Consulta: 08 de julio de 2015].

VELA, José Antonio (2001): "Los estudios de la audiencia: una visión crítica desde la economía política". Revista Latina de Comunicación Social, 43: http://goo.gl/PD5afi [Consulta: 14 de agosto de 2015].

WEBLEY, Kayla (2010): "How the Nixon-Kennedy Debate Changed the World", en Time, 23 de septiembre de 2010: http://goo.gl/IjP5f5 [Consulta: 1 de agosto de 2015].

Jorge Gallardo-Camacho es doctor en Comunicación Audiovisual por la Universidad de Málaga y profesor de la Universidad Camilo José Cela (Facultad de Comunicación Departamento de Comunicación Audiovisual)

Eva Lavín es doctora en Comunicación Audiovisual y profesora de la Universidad Camilo José Cela (Facultad de Comunicación, Departamento de Comunicación Audiovisual) 